\title{
EFFECT OF THE ION STABILITY ON THE FATE OF INTERMEDIATE ION/NEUTRAL COMPLEXES
}

\author{
HANS-FRIEDRICH GRÜTZMACHER*, GERHARD THIELKING, \\ DORIS WITTNEBEN and DIRK EIKENBERG
}

Fakultät für Chemie der Universität Bielefeld, Postfach 8640, Universitätsstraße, D-4800 Bielefeld (F.R.G.)

(First received 22 January 1990; in final form 1 March 1990)

\begin{abstract}
Carbonyl protonated aromatic ketones a-i $\left[\mathrm{R}-\mathrm{C}\left(\mathrm{OH}^{+}\right)-\mathrm{C}_{6} \mathrm{H}_{4} \mathrm{CH}_{2} \mathrm{OCH}_{3}\right]$ containing a 4-methoxymethylphenyl group and $\mathrm{R}=\mathrm{H}, \mathrm{CH}_{3}, \mathrm{C}_{2} \mathrm{H}_{5}, n-\mathrm{C}_{3} \mathrm{H}_{7}, \mathrm{i}-\mathrm{C}_{3} \mathrm{H}_{7}, \mathrm{C}_{6} \mathrm{H}_{5}, 4-\mathrm{CH}_{3} \mathrm{OC}_{6} \mathrm{H}_{4}$, $4-\mathrm{CF}_{3} \mathrm{C}_{6} \mathrm{H}_{4}$, and $\mathrm{CF}_{3}$, respectively, are generated in the ion source by $70 \mathrm{eV}$ electron impact induced dissociation of the benzylic alcohols 1-10. The ions a-i may dissociate to yield acylium ions of different stability. The spontaneous fragmentations of metastable $\mathbf{a}-\mathbf{i}$ have been studied by mass-analysed ion kinetic energy spectrometry and the reactions observed generally agree with the mechanisms suggested before for similar protonated aromatic ketones. In particular, these ions form intermediate ion/neutral complexes consisting of an acylium ion $\mathrm{RCO}^{+}$and a benzyl methyl ether molecule, and fragment by an internal ion/molecule reaction generating $\mathrm{C}_{7} \mathrm{H}_{7}^{+}$and $\mathrm{RCOOCH}_{3}$ as well as by a direct dissociation releasing $\mathrm{RCO}^{+}$. By varying $\mathrm{R}$ it is shown that the competition between these fragmentations of metastable ions depends strongly on the stability of $\mathrm{RCO}^{+}$. In the case of carbonyl protonated benzophenones the proton migrates predominantly to the more basic (substituted) phenyl group. Labelling experiments show that a proton transfer between the two phenyl groups of the protonated benzophenones does not occur.
\end{abstract}

\section{INTRODUCTION}

The role of ion/neutral complexes as intermediates of unimolecular fragmentations of organic ions in a mass spectrometer is well recognized [1]. In particular the fragmentations of metastable ions at low internal energies are mediated by these complexes. Obviously the long-range ion-dipole and ioninduced dipole interactions slow down a direct dissociation into the components, and especially at low internal energies the reacting system may cross on the potential surface from the direct dissociation channel to an energetically more favourable reaction channel giving rise to stable products by an internal ion/molecule reaction [2]. Considering the competition between a

* Author to whom correspondence should be addressed. 


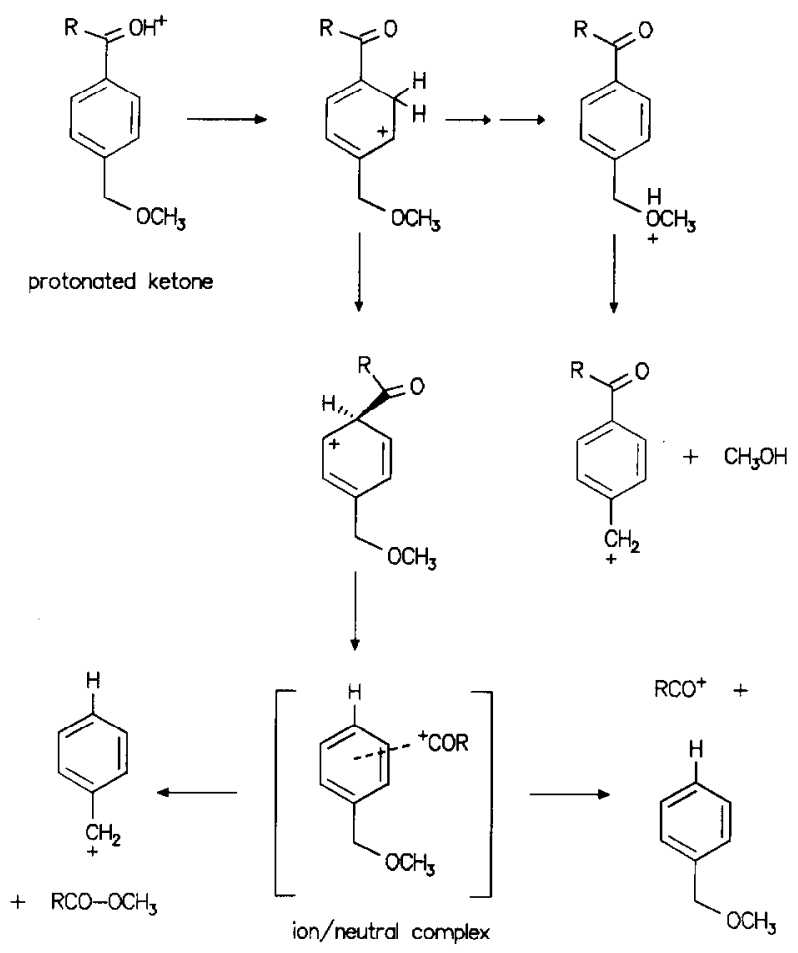

Scheme 1

direct dissociation and other fragmentation modes of an ion/neutral complex, a prerequisite for fragmentation by an internal ion/molecule reaction is not only a favourable reaction energy but also a small activation energy to guarantee a fragmentation pathway totally preferred to direct dissociation. This explains why internal ion/molecule reactions corresponding to the transfer of a proton, a hydrogen atom and a hydride ion, respectively, and requiring only a small activation energy are frequently observed in intermediate ion/neutral complexes $[1,3]$. However, fragmentations mediated by other types of internal ion/molecule reactions are also observed [1,3a,4]. Recently we have shown that carbonyl protonated benzaldehydes [5], acetophenones [6], and other aromatic aldehydes and ketones [7] substituted at the aromatic ring by a methoxymethyl group fragment via intermediate ion/neutral complexes consisting of an acylium ion and a benzyl methyl ether molecule (Scheme 1).

The complexes are generated by a proton migration from the carbonyl group to the aromatic ring followed by a protolytic bond cleavage to release the formyl cation $\left(\mathrm{R}=\mathrm{H}\right.$ ) and acetyl cation $\left(\mathrm{R}=\mathrm{CH}_{3}\right)$, respectively. A very interesting fragmentation of the resulting complex is a transfer of the acylium 
ion across the aromatic ring to the ether group where the ion reacts by an electrophilic ether cleavage to lose an arylmethyl cation and a neutral ester molecule. The initial proton transfer very probably needs a substantial activation energy as suggested by MNDO calculations [7] and thus gives rise to an ion/neutral complex excited by chemical activation. This probably enables the acylium ion to traverse large molecular distances within the complex $[7,8]$ and to surmount the activation barrier of the ether cleavage reaction. However, the excess energy within a loosely bound ion/neutral complex [2] and the activation energy of an internal ion/molecule reaction tolerable in competition to a direct dissociation depends of course on the reaction energy of this latter process and in particular on the thermodynamic stability of the ionic component. As a consequence an intermediate ion/neutral complex formed from a metastable precursor and containing an unstable ionic component may fragment by internal ion/molecule reactions while an analogous complex containing a stable ion prefers direct dissociation. In fact, the $\mathrm{CH}_{3} \mathrm{CO}^{+}$ cations give rise to one of the prominent peaks in the $70 \mathrm{eV}$ mass spectra of the 2-aryl propan-2-ols used as precursors for the protonated methyl aryl ketones [6-8] and is normally the base peak in the CA spectra of the protonated ketones [9]. This shows clearly that direct dissociation prevails for ion/neutral complexes with sufficient excess energy. To test these effects, in particular the influence of the stability of the acylium ion on the reaction of intermediate acylium ion/benzyl methyl ether complexes, the fragmentations of the carbonyl protonated aromatic ketones $\mathbf{c}-\mathbf{i}$ have now been studied in addition to the carbonyl protonated species $\mathbf{a}[5]$ and $\mathbf{b}[6]$.

\section{EXPERIMENTAL}

The mass spectrometric measurements were performed with a double focusing mass spectrometer VG ZAB-2F (VG Analytical Ltd., Wythenshawe, Manchester M2 9LE, Gt. Britain) equipped with a combined EI-CI ion source using the following conditions: electron energy $70 \mathrm{eV}$, electron trap current $50 \mu \mathrm{A}$, accelerating voltage $6 \mathrm{kV}$, ion source temperature ca. $180^{\circ} \mathrm{C}$, sample admission to the ion source either by a heated direct inlet system with a variable leak valve or with a direct sample probe at ambient temperature. The reactions of the metastable ions in the second field free region (2nd FFR) behind the magnet were studied by focusing the relevant ion magnetically into the 2nd FFR and detecting the product of the spontaneous fragmentations by scanning the deflection voltage of the electrostatic analyser (mass-analysed ion kinetic energy (MIKE) spectra). The ions were detected with an electron multiplier and the MIKE spectra were recorded on a pen recorder. The MIKE spectra given in the tables are the mean of at least three spectra.

The benzylic alcohols 1-10 (Scheme 2 ) used as precursors of the protonated 

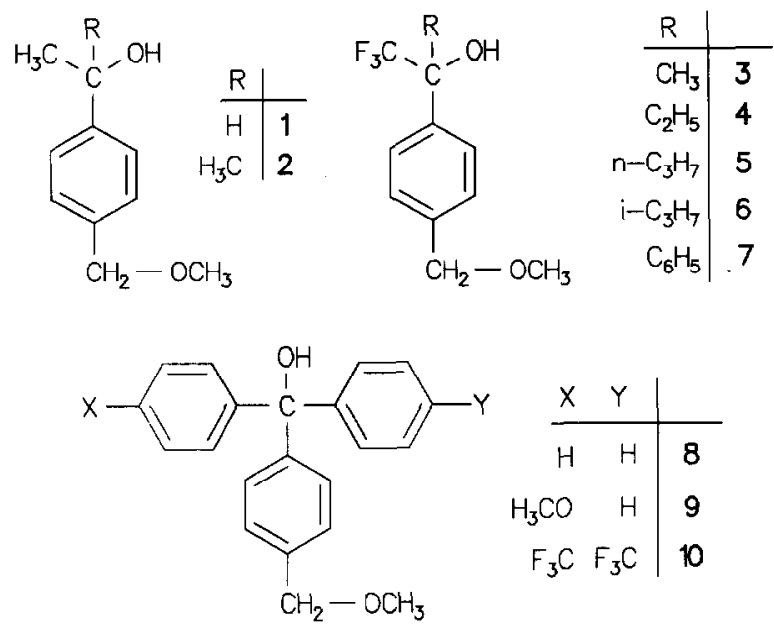

Scheme 2

carbonyl compounds were prepared from 4-methoxymethylacetophenone [5,6] (for 1 and 2), 1,1,1-trifluoro-4-methoxymethylacetophenone (for 3-7), and 4-methoxymethylbenzophenone (for 8-10), respectively, by treatment with the appropriate Grignard reagent using standard techniques of organic chemistry. The compounds were purified by fractional distillation at reduced pressure and column chromatography as convenient. The purity of all compounds was controlled by thin layer chromatography and their structures were verified by ${ }^{1} \mathrm{H}$ NMR spectroscopy.

The alcohols deuterated at the hydroxy group were obtained by treatment of 1-10 with excess $\mathrm{D}_{2} \mathrm{O}$ shortly before the mass spectrometric investigation and measured as usual in the presence of some $\mathrm{D}_{2} \mathrm{O}$ in the ion source.

The deuterated derivatives of 8 and 9 carrying six D atoms at the methoxymethylated phenyl group were synthesized from 4-(methoxydideuteromethyl)-tetradeutcrobenzophenone and the appropriate Grignard reagent. The starting material for the hexadeuterated benzophenone was octadeuterotoluene commercially available which was converted into heptadeutero-para-toluic acid by acetylation and subsequent haloform oxidation of the para-acetyl group. The toluic acid was transformed into the acid chloride which was used in a Friedel-Crafts aroylation of benzene to yield 4-trideuteromethyl-tetradeuterobenzophenone. This was brominated at the trideuteromethyl group using $N$-bromosuccinimide and converted into the 4-(methoxy-dideuteromethyl)-tetradeuterobenzophenone by treatment with $\mathrm{CH}_{3} \mathrm{ONa} / \mathrm{CH}_{3} \mathrm{OH}$. 


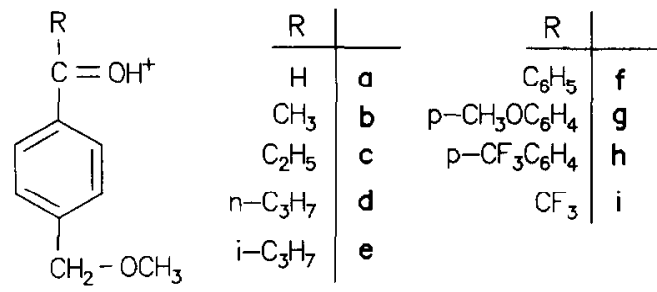

Scheme 3

\section{RESULTS AND DISCUSSION}

The ions a-i (Scheme 3) were generated in the ion source by electron impact induced dissociation of the alcohols 1-10 (Scheme 2) and their spontaneous reactions were studied by MIKE spectrometry. The MIKE spectra are shown in Table 1. (Some of the ions $\mathbf{b}-\mathbf{g}$ may be generated from more than one precursor due to competing $\alpha$-cleavages of the molecular ions. The MIKE spectra of a specific ion from different precursors agree reasonably. The largest variations are observed for the relative intensity of the loss of methanol.)

The fragmentations common to all metastable ions a-i agree with mechanisms discussed before [5-7]. In particular the MIKE spectra of deuterated derivatives of $\mathbf{a}-\mathbf{i}$ carrying initially an OD group reveal an exchange of this $\mathrm{D}$ atom with the $\mathrm{H}$ atoms at the benzene ring before the loss of methanol, while no $\mathrm{H} / \mathrm{D}$ exchange accompanies the other fragmentations. That is, the $\mathrm{D}$ atom is always retained by the ionic fragment for the loss of $\mathrm{CH}_{2} \mathrm{O}$ and the ester $\mathrm{RCOOCH}_{3}$, respectively, and remains with the neutral fragment during the formation of $\mathrm{RCO}^{+}$and $\mathrm{CH}_{3} \mathrm{OCH}_{2}^{+}$, respectively.

The fragmentations of interest in the present context are the elimination of an ester molecule $\mathrm{RCOOCH}_{3}$ formed by the internal ion/molecule reaction of

TABLE 1

MIKE spectra of protonated aromatic carbonyl compounds

\begin{tabular}{lrrrrrrrrr}
\hline $\begin{array}{l}\text { Fragmentation/ } \\
\text { fragment ion }\end{array}$ & a & b & c & d & e & f & g & h & i \\
\hline$-\mathrm{CH}_{2} \mathrm{O}$ & 2 & 3 & 4 & 5 & 4 & 9 & 9 & 0 & 0 \\
$-\mathrm{CH}_{3} \mathrm{OH}$ & 69 & 40 & 30 & 24 & 74 & 20 & 17 & 63 & 49 \\
$-\mathrm{RCOOCH}_{3}$ & 8 & 33 & 31 & 26 & 4 & 4 & 0 & 7 & 0 \\
$\mathrm{RCO}^{+}$ & 0 & 6 & 13 & 22 & 0 & 40 & 34 & 27 & 1 \\
$\mathrm{CH}_{3} \mathrm{OCH}_{2}^{+}$ & 20 & 17 & 22 & 23 & 17 & 13 & 3 & 3 & 16 \\
Other & & & & & & a & b & & c
\end{tabular}

${ }^{a}-\mathrm{C}_{6} \mathrm{H}_{6}, 13 \%,{ }^{b}-\mathrm{CH}_{3} \mathrm{O}-\mathrm{C}_{6} \mathrm{H}_{5}, 32 \% .{ }^{\mathrm{c}}-\mathrm{CHF}_{3}, 27 \% ;-\mathrm{CH}_{3}, 4 \% ;-\mathrm{OH}, 3 \%$. 
TABLE 2

Hydride ion affinity ( $\mathrm{HIA})\left(\mathrm{kJ} \mathrm{mol}^{-1}\right)$ of $\mathrm{RCO}^{+}$and intensity ratio $q\left(=\mathrm{RCO}^{+} / \mathrm{C}_{7} \mathrm{H}_{7}^{+}\right)$in the MIKE spectra of ions $\mathbf{a}-\mathbf{h}$

\begin{tabular}{lccccccc}
\hline $\begin{array}{l}\text { Ion } \\
\begin{array}{l}\text { Parameter } \\
\left(\mathrm{kJ} \mathrm{mol}^{-1}\right)\end{array}\end{array}$ & a & b & c & d & f & g & h \\
\hline$\Delta H_{\mathrm{f}}\left(\mathrm{RCO}^{+}\right)^{\mathrm{a}}$ & 825.6 & 653 & 591 & $<620^{\mathrm{b}}$ & 705 & & \\
$\Delta H_{\mathrm{f}}\left(\mathrm{RCHO}^{\mathrm{c}}\right.$ & -108.6 & -166.1 & -185.6 & -204.8 & -36.7 & & \\
$\left.\mathrm{HIACO}^{+}\right)^{\mathrm{d}}$ & 1079 & 964 & 922 & $<970$ & 877 & & \\
$q$ & $<0.1$ & 0.2 & 0.4 & 0.8 & 10 & $>34$ & 3.9 \\
\hline
\end{tabular}

${ }^{a}$ Ref. 12.

${ }^{\mathrm{b}}$ This value is derived from the $\mathrm{AE}=10.03 \mathrm{eV}$ and $\Delta H_{\mathrm{f}}\left(\mathrm{CH}_{3}\right)=142.3 \mathrm{~kJ} \mathrm{~mol}^{-1}[14]$ and is probably an upper limit.

'Ref. 14.

${ }^{\mathrm{d}} \mathrm{HIA}=\Delta H_{\mathrm{f}}\left(\mathrm{RCO}^{+}\right)+\Delta H_{\mathrm{f}}\left(\mathrm{H}^{-}\right)-\Delta H_{\mathrm{f}}(\mathrm{RCHO})$, using $\Delta H_{\mathrm{f}}\left(\mathrm{H}^{-}\right)=145 \mathrm{~kJ} \mathrm{~mol}^{-1}$.

the intermediate complex and the dissociation of the complex liberating the acylium ion $\mathrm{RCO}^{+}$. A useful scale for the stability of a carbenium ion is the hydride ion affinity (HIA) [10], which decreases with increasing stability of the ion. The $\mathrm{HIA}\left(\mathrm{RCO}^{+}\right)$are shown in Table 2. As a general trend the data of Table 1 show an increase of the relative abundance of $\mathrm{RCO}^{+}$in the MIKE spectra of $\mathbf{a}-\mathbf{i}$ with the stability of the acylium ion. Thus, the unstable ions $\mathrm{HCO}^{+}$and $\mathrm{CF}_{3} \mathrm{CO}^{+}$are not detected in the MIKE spectra of a and $\mathbf{i}$, respectively, while the stable benzoyl ion and its substituted derivatives give rise to intense signals in the spectra of $\mathbf{f}-\mathbf{h}$.

The MIKE spectrum of ion $\mathbf{e}$ is exceptional since the peak of the expected isobutyryl ion $\left(\mathrm{CH}_{3}\right)_{2} \mathrm{CHCO}^{+}$is not detected in contrast to the disinct peak of the $n$-butyryl ions $\mathrm{CH}_{3} \mathrm{CH}_{2} \mathrm{CH}_{2} \mathrm{CO}^{+}$in the case of ion $\mathrm{d}$. Furthermore the signal due to the loss of the ester $\left(\mathrm{CH}_{3}\right)_{2} \mathrm{CHCOOCH}_{3}$ is very small and that for the loss of $\mathrm{CH}_{3} \mathrm{OH}$ is unusually large in the MIKE spectrum of $\mathbf{e}$. However, the $\mathrm{H} / \mathrm{D}$ exchange rates accompanying the elimination of methanol in the MIKE spectra of the O-deuterated derivatives of $\mathbf{d}$ and $\mathbf{e}$ are rather similar $\left(-\mathrm{CH}_{3} \mathrm{OH} /-\mathrm{CH}_{3} \mathrm{OD} 68: 32\right.$ for $\mathbf{d}$ and $73: 27$ for e), so there is no evidence for a special mechanism for the loss of methanol from e. Obviously the formation of the isobutyryl cation by the protolytic bond cleavage subsequent to the initial proton transfer is suppressed with the effect that the elimination of methanol gains in intensity; however, the reason for this (steric effects?) remains unclear.

The ion $\mathbf{i}$ containing a protonated trifluoroacetyl group also represents a special case. The MIKE spectrum of $\mathbf{i}$ exhibits a barely detectable peak for $\mathrm{CF}_{3} \mathrm{CO}^{+}$and no peak for the elimination of $\mathrm{CF}_{3} \mathrm{COOCH}_{3}$ and $\mathrm{CH}_{2} \mathrm{O}$, respectively, while the elimination of $\mathrm{CH}_{3} \mathrm{OH}$ and the formation of $\mathrm{CH}_{3} \mathrm{OCH}_{2}^{+}$are 
observed with typical intensities in addition to an abundant loss of $\mathrm{CF}_{3} \mathrm{H}$. The electron-withdrawing $\mathrm{CF}_{3}$ substituent causes the carbonyl group of 1,1,1-trifluoroacetophenone to be the least basic one in the series of aromatic ketones studied. Hence one would expect an easy migration of the proton at the carbonyl group of $\mathbf{i}$ to the aromatic ring. In fact the loss of methanol is accompanied by the usual $\mathrm{H} / \mathrm{D}$ exchange $\left(-\mathrm{CH}_{3} \mathrm{OH} /-\mathrm{CH}_{3} \mathrm{OD} 79: 21\right)$ in the case of the $\mathrm{O}$-deuterated derivative. The release of $\mathrm{CH}_{3} \mathrm{OCH}_{2}^{+}$by a protolytic bond cleavage of the other substituent at the aromatic ring is also observed, but not the methylation of the carbonyl group resulting in the ion $\left[\mathrm{i}-\mathrm{CH}_{2} \mathrm{O}\right]$ [4]. Obviously this reaction is not accessible by metastable ions i because the small methyl cation affinity of the trifluoroacetyl group makes this reaction energetically unfavourable. Similarly, $\mathrm{CF}_{3} \mathrm{CO}^{+}$is not released by protolytic bond cleavage within $\mathbf{i}$ because of the large reaction energy needed to produce this unstable reaction product. Instead, elimination of $\mathrm{CF}_{3} \mathrm{H}$ is observed, and the loss of $\mathrm{CF}_{3} \mathrm{H}$ and $\mathrm{CF}_{3} \mathrm{D}$ in a ratio of roughly $2: 1$ from the O-deuterated $i$ reveals $H / D$ exchanges between the carbonyl $D$ and the $H$ atoms of the aromatic ring preceding this process as in the case of the elimination of methanol. However, the H/D exchanges accompanying the elimination of $\mathrm{CF}_{3} \mathrm{H}$ are distinctly less than in the latter process.

The competition between the direct dissociation and an internal ion/ molecule reaction of the acylium ion/benzyl methyl ether complex is seen more clearly from the intensity ratio $\mathbf{q}$ of the free acylium ion and the product ion $\mathrm{C}_{7} \mathrm{H}_{7}^{+}$of the ester elimination reaction in the MIKE spectra of $\mathbf{a}-\mathbf{h}$ (Table 2). The ratio $q$ increases with decreasing HIA from a value of 0.2 for the acetyl cation (ion b) to a value of 10 for the benzoyl cation (ion $\mathbf{f}$ ), in agreement with the behaviour expected for an ion/neutral complex. Similarly, the substituent effect on the stability of the benzoyl ion is reflected clearly in the ratio $q$ rising from 3.9 in the case of $p-\mathrm{CF}_{3} \mathrm{C}_{6} \mathrm{H}_{4} \mathrm{CO}^{+}$(ion h) with an electron-withdrawing substituent to $>34$ for $p-\mathrm{CH}_{3} \mathrm{OC}_{6} \mathrm{H}_{4} \mathrm{CO}^{+}$(ion $\mathrm{g}$ ) with an electron-donating group. In fact no signal for the elimination of an ester molecule is observed in the latter case.

The MIKE spectra of the protonated benzophenones $\mathbf{f}-\mathbf{h}$ warrant some additional comments. The proton at the carbonyl group conceivably migrates to either of the substituted aromatic rings at both sides of the carbonyl group, depending on their respective proton affinity. A proton migration to the methoxymethyl substituted ring gives rise subsequently to the fragmentations discussed above and eventually to a benzoyl cation consisting of the other phenyl group. Similarly, an initial proton migration to the other phenyl group of $\mathbf{f}-\mathbf{h}$ followed by a protolytic bond cleavage should result in the loss of a (substituted) benzene molecule and the formation of $\mathrm{CH}_{3} \mathrm{OCH}_{2}-\mathrm{C}_{6} \mathrm{H}_{4} \mathrm{CO}^{+}$, $m / z 149$ (Scheme 4). In fact the MIKE spectrum of $f$ exhibits this signal for the loss of $\mathrm{C}_{6} \mathrm{H}_{6}$ with $13 \%$ relative intensity. The $\mathrm{CH}_{3} \mathrm{O}$ substituent at the second phenyl ring of $\mathbf{g}$ enhances the proton affinity of, and therefore the 


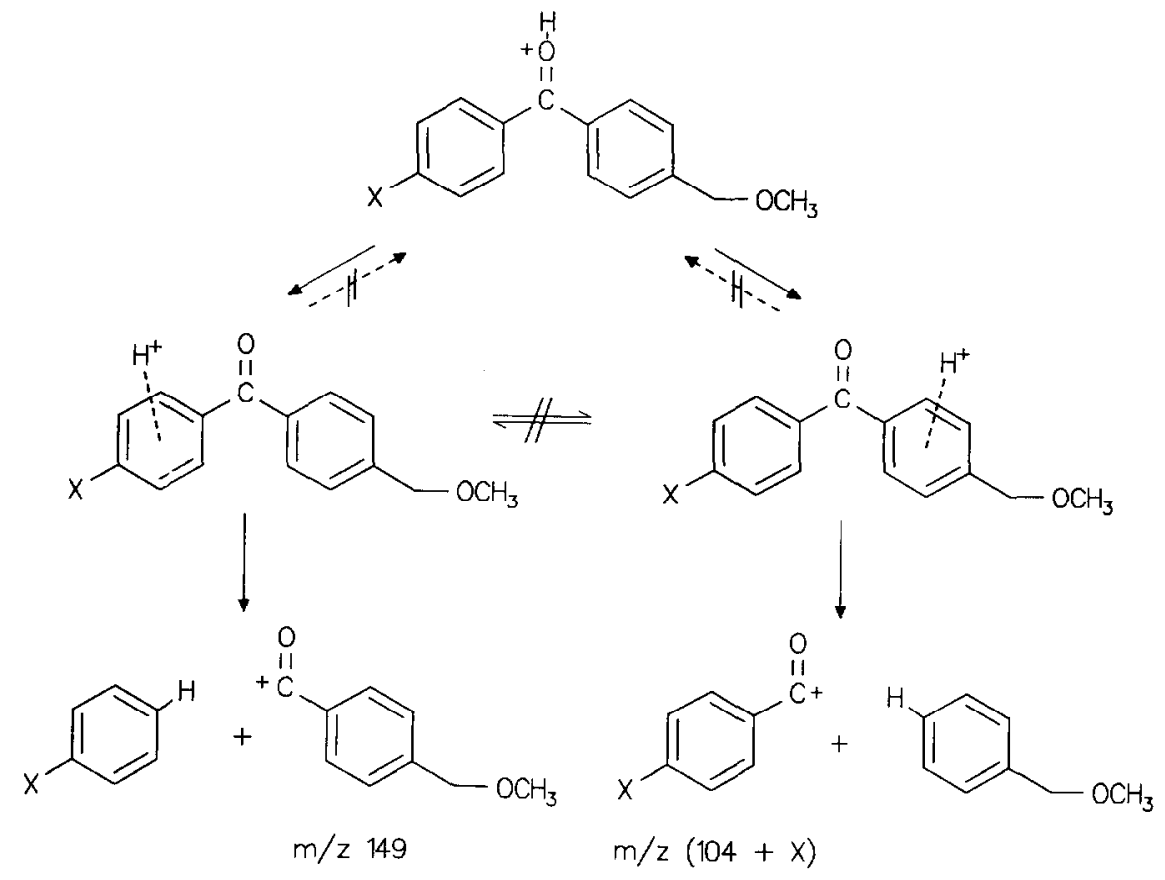

Scheme 4

initial proton migration to, this ring. Thus, the signal at $m / z 149$ due to the loss of $\mathrm{CH}_{3} \mathrm{O}-\mathrm{C}_{6} \mathrm{H}_{5}$ increases to $32 \%$ in the MIKE spectrum of $\mathrm{g}$. In contrast, the $\mathrm{CF}_{3}$ substituent of $\mathbf{h}$ makes the second phenyl ring less basic and only the final products of a proton migration to the other aromatic ring of $\mathbf{h}$ are observed, i.e., loss of $\mathrm{CH}_{3} \mathrm{OCH}_{2}-\mathrm{C}_{6} \mathrm{H}_{5}$ and formation of $\mathrm{CF}_{3}-\mathrm{C}_{6} \mathrm{H}_{4} \mathrm{CO}^{+}$in spite of the unfavourable heat of formation of this latter ion.

It seemed likely that the proton (or deuteron) interchanges its position at the carbonyl group and at the various positions at both aromatic rings of $\mathbf{f}-\mathbf{h}$ before the fragmentations. The proton transfer between the two phenyl groups of protonated $\alpha, \omega$-diphenylalkanes and related compounds has been extensively studied by us [11] and usually a fast inter- and intra-annular proton exchange yielding complete "scrambling" is observed. The exceptions are protonated diphenylmethanes which exhibit a slow but nevertheless distinct inter-annular proton exchange [1 le,f]. The carbon skeletons of diphenylmethane and benzophenone are identical and one expects a similar proton exchange rate for both protonated molecules. In this case any $D$ atoms originally present at one of the phenyl groups of the benzophenone should eventually also appear in the fragments containing the other phenyl group. However, the MIKE spectra of deuterated derivatives of $f$ and $g$ carrying $6 \mathrm{D}$ 
atoms at the methoxymethylphenyl moiety exhibit only peaks at $m / z 105$ and $m / z 155$, due to $\mathrm{C}_{6} \mathrm{H}_{5} \mathrm{CO}^{+}$and $\mathrm{CH}_{3} \mathrm{OCD}_{2}-\mathrm{C}_{6} \mathrm{D}_{4} \mathrm{CO}^{+}$, respectively, and at $m / z$ 173 and $m / z 155$, the former due to $\mathrm{CF}_{3}-\mathrm{C}_{6} \mathrm{H}_{4} \mathrm{CO}^{+}$. Thus, any "scrambling" between the $\mathrm{H}$ atoms at one ring and the $\mathrm{D}$ atoms at the other one does not occur, and the direct inter-annular proton exchange rate of a protonated benzophenone must be distinctly smaller than those of protonated $\alpha, \omega$ diphenylalkanes [11]. Obviously the fragmentations by protolytic bond cleavage subsequent to the initial proton transfer from the carbonyl group are also much faster than a back migration of a proton to the carbonyl group and eventually to the other aromatic ring. This agrees with the assumption that the proton transfer from the carbonyl group to an aromatic ring and vice versa requires a considerable activation energy [6] so that the ions formed by this proton shift decompose quickly because of a chemical activation.

\section{ACKNOWLEDGEMENTS}

We thank the Deutsche Forschungsgemeinschaft and the Fond der Chemischen Industrie for financial support of this work.

\section{REFERENCES}

1 For a review see: D.J. McAdoo, Mass Spectrom. Rev., 7 (1988) 363.

2 E.L. Chronister and T.H. Morton, J. Am. Chem. Soc., 112 (1990) 133.

3 (a) T.H. Morton, Tetrahedron, 38 (1982) 3195.

(b) R.W. Kondrat and T.H. Morton, Org. Mass Spectrom., 23 (1988) 555.

(c) S. Hammerum and H.E. Audier, Adv. Mass Spectrom., Part A, 11 (1989) 894.

4 H.-Fr. Grützmacher and G. Thielking, Org. Mass Spectrom., 23 (1988) 397.

5 U. Filges and H.-Fr. Grützmacher, Org. Mass Spectrom., 21 (1986) 673.

6 U. Filges and H.-Fr. Grützmacher, Org. Mass Spectrom., 22 (1987) 444.

7 U. Filges and H.-Fr. Grützmacher, Int. J. Mass Spectrom. Ion Processes, 83 (1988) 111

8 H.-Fr. Grützmacher, U. Filges and G. Thielking, to be published.

9 G. Thielking, Diplomarbeit, Universität Bielefeld, 1987.

10 D.H. Aue and M.T. Bowers, in M.T. Bowers (Ed.), Gas Phase Ion Chemistry, Vol. 2, Academic Press, London, 1979, Chap. 9.

11 (a) D. Kuck, W. Bäther aṇd H.-Fr. Grützmacher, J. Am. Chem. Soc., 101 (1979) 7154.

(b) W. Bäther, D. Kuck and H.-Fr. Grützmacher, Org. Mass Spectrom., 20 (1985) 572.

(c) W. Bäther, D. Kuck and H.-Fr. Grützmacher, Org. Mass Spectrom., 20 (1985) 589.

(d) W. Bäther, D. Kuck and H.-Fr. Grützmacher, Int. J. Mass Spectrom. Ion Processes, 67 (1985) 75.

(e) D. Kuck, W. Bäther, Org. Mass Spectrom., 21 (1986) 451.

(f) D. Kuck. Int. J. Mass Spectrom. Ion Phys., 47 (1983) 499.

(g) D. Kuck and U. Fastabend, Adv. Mass Spectrom., Part A, 11, (1989) 906.

(h) D. Kuck, D. Thölmann and H.-Fr. Grützmacher, J. Chem. Soc., Perkin Trans 2., (1990) 251.

12 S.G. Lias, J.E. Bartmess, J.F. Liebman, J.L. Holmes, R. Levin and W.G. Mallard, J. Phys. Chem. Ref. Data, 17 (Suppl. 1) (1988). 
13 H.M. Rosenstock, K. Draxl, B.W. Steiner and J.T. Herron, J. Phys. Chem. Ref. Data, 6 (Suppl. 1) (1977).

14 J.B. Pedley, R.D. Naylor and S.P. Kirby, Thermochemical Data on Organic Compounds, Chapman and Hall, London, 1986. 